Rev. Biol. Trop., 48(2/3): 425-438, 2000

www.ucr.ac.cr www.ots.ac.cr www.ots.duke.edu

\title{
Evaluación de la riqueza de especies de peces en las lagunas costeras mexicanas: Estudio de un caso en el Golfo de México
}

\author{
Marco Aurelio Pérez-Hernández ${ }^{1}$ y Roberto E. Torres-Orozco B. ${ }^{2}$ \\ Departamentos de Biología ${ }^{1}$ e Hidrobiología ${ }^{2}$, D.C.B.S. Universidad Autónoma Metropolitana - Iztapalapa. \\ Apdo. Postal 55-535, Código Postal 09340, México, D.F. Fax: (52) 5804-4688. e-mail: maph @xanum.uam.mx
}

Recibido 10-VI-1999. Corregido 10-II-2000. Aceptado 23-III-2000.

\begin{abstract}
We analyze the origin of knowledge about fish species richness in the Tuxpan-Tampamachoco estuarine system, in Veracuz, México. A complete inventory of the fish species known to date for this system $(\mathrm{N}=179)$ was elaborated from published lists and from sampling seagrass meadows of Tampamachoco Lagoon, which yielded 14 previously unknown species. When compared, the different lists showed a low similarity that may reflect differences in sampling methods and collecting strategies. Current data suggest that fish species richness in Mexican coastal lagoons (Gulf of Mexico) is not related with lagoon surface area, as has been suggested, but with the number of inventories available for each lagoon, being these a reflection of the sampling effort. A sampling design for the assessment of fish species richness in estuarine systems should consider: a) using the highest possible variety of sampling fishing gears, b) collecting in all microhabitat types and c) the preference of bimonthly or quarterly samplings for two or more years over monthly samplings in a single year.
\end{abstract}

Key words: Fishes, species richness, coastal lagoons, estuaries, Gulf of Mexico.

En 1978, Castro-Aguirre publicó el primer inventario de los peces estuarino-lagunares de México. Basándose en una minuciosa revisión bibliográfica y en el estudio de varias colecciones científicas, registró más de 350 especies de peces marinos que penetran a las aguas continentales mexicanas. En fechas más recientes, Fuentes-Mata (1991) estimó en cerca de 450 especies la riqueza íctica de los estuarios y las lagunas costeras de México, señalando que ésta era una de las más altas registradas en las zonas tropicales del mundo. No obstante, esta cifra podría ser aún mayor, pues tan sólo en las lagunas costeras del Golfo de México Reséndez y Kobelkowsky (1991) documentaron la presencia de 318 especies de peces.
Los peces que habitan en el sistema formado por el estuario del Río Tuxpan y la Laguna de Tampamachoco, en el estado mexicano de Veracruz, han sido objeto de numerosas investigaciones; sin embargo, las publicaciones al respecto son relativamente escasas. Los trabajos que abordan el estudio de la comunidad de peces y ofrecen listados de especies son básicamente siete: Chávez (1972), Kobelkowsky (1985), Castro-Aguirre et al. (1986) y Castro-Aguirre (1986), Sánchez-Rueda (1986), López-López et al. (1991), y Cota y Santiago (1994).

En este trabajo se presenta la lista más completa disponible hasta la fecha de los peces del sistema Tuxpan-Tampamachoco. Ésta incluye, además de los registros previamente 
publicados, información derivada de colectas propias que nos permitieron reconocer la presencia de 85 especies de peces, 14 de las cuales son primeros registros en la zona. Dado que éste es uno de los ambientes mejor conocidos del Golfo de México, el estudio del caso, basado en el análisis cronológico y comparativo de los listados de especies de peces generados por distintos autores, incluido el nuestro, ofrece información relevante sobre la manera en la que se ha venido conformando el conocimiento de la ictiofauna estuarino-lagunar de México. Los resultados de este análisis permiten proponer algunas generalizaciones aplicables a las lagunas costeras del Golfo de México, así como plantear un conjunto de recomendaciones metodológicas para la evaluación de la riqueza de especies de peces estuarinos.

\section{MATERIALES Y MÉTODOS}

El listado elaborado por nosotros tuvo como base los registros obtenidos en un programa de recolecciones mensuales, desarrollado entre mayo de 1990 y junio de 1991, en las áreas someras con pastos marinos de la margen poniente de la Laguna de Tampamachoco, empleando un chinchorro de malla fina (ver Cuadro 1). Esta lista se integró con las previamente publicadas para formar un solo inventario combinado. Las distintas listas se compararon entre sí aplicando el índice de similitud de Jaccard y se analizaron las estrategias de muestreo empleadas en cada caso.

Se exploraron, mediante análisis de correlación simple, las relaciones que guarda la riqueza de especies de peces registrada en 13 lagunas costeras del Golfo de México (Fig. 1) con su área superficial y con el esfuerzo de recolecta. Dada la dificultad para obtener en todos los casos información precisa de esta última variable, se consideró conveniente estandarizarla suponiendo que el número de trabajos ictiológicos, en general, y de inventarios ictiofaunísticos, en particular, disponibles para cada laguna, podría representar una medida adecuada del esfuerzo de pesca invertido en el propósito de documentar su riqueza íctica.

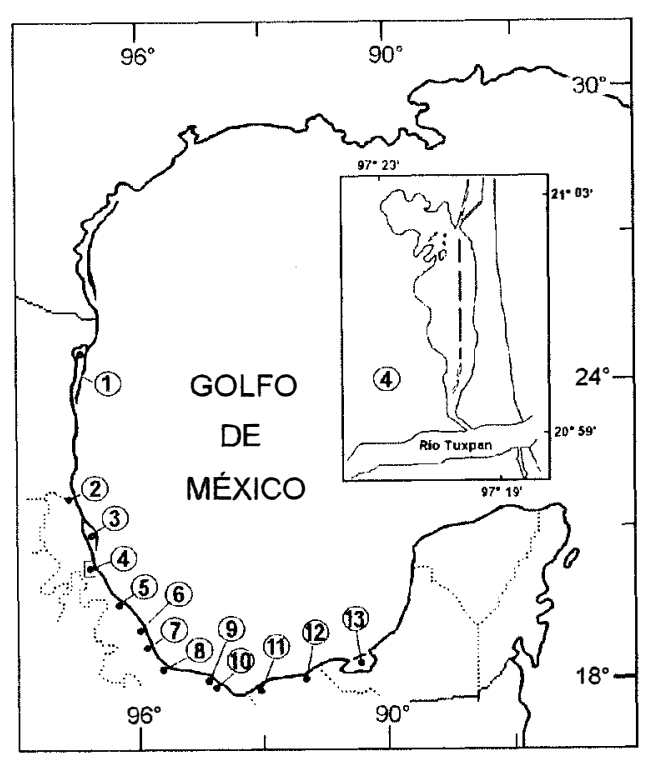

Fig. 1. Localización del sistema estuarino-lagunar de Tuxpan-Tampamachoco y de las principales lagunas costeras del Golfo de México. 1) Laguna Madre, 2) Laguna de Pueblo Viejo, 3) Laguna de Tamiahua, 4) Sistema TuxpanTampamachoco, 5) Laguna Grande, 6) Laguna de la Mancha, 7) Laguna de Mandinga, 8) Laguna de Alvarado, 9) Laguna de Sontecomapan, 10) Laguna del Ostión, 11) Sistema Carmen-Machona, 12) Laguna de Mecoacán, 13) Laguna de Términos.

Fig. 1. Location of the Tuxpan-Tampamachoco estuarine system and main Mexican coastal lagoons of the Gulf of Mexico. 


\section{CUADRO 1}

Sinopsis de las estrategias de recolecta utilizadas y el número de especies de peces registradas por distintos autores en el sistema estuarino-lagunar de Tuxpan-Tampamachoco.

Table 1. Synopsis of the used sampling strategies and number of fish species recorded by different authors in the TuxpanTmpamachoco estuarine system.

\begin{tabular}{|c|c|c|c|c|c|c|}
\hline Autor & $\begin{array}{l}\text { Período } \\
\text { de estudio } \\
\text { mes/año }\end{array}$ & Arte de pesca & $\begin{array}{l}\text { Intensidad } \\
\text { de muestreo }\end{array}$ & $\begin{array}{l}\text { Sitios de } \\
\text { recolecta }\end{array}$ & $\begin{array}{l}\text { No. de } \\
\text { Especies } \\
\text { y }(\%)^{1}\end{array}$ & $\begin{array}{c}\text { Registros } \\
\text { nuevos }\end{array}$ \\
\hline $\begin{array}{l}\text { Chávez } \\
1972 .\end{array}$ & $\begin{array}{c}12 / 64 \\
\dot{a} \\
03 / 66\end{array}$ & $\begin{array}{l}\text { Red de arrastre } \\
\text { (boca } 3.0 \mathrm{~m} \text { ) }\end{array}$ & $\begin{array}{l}\text { Bimestral } \\
12 \text { muestreos } \\
13 \text { estaciones } \\
97 \text { lances }\end{array}$ & $\begin{array}{l}\text { Estuario del río y } \\
\text { canalde la laguna; } \\
\text { profundos }\end{array}$ & $\begin{array}{c}61 \\
(34.1)\end{array}$ & $61^{2}$ \\
\hline $\begin{array}{l}\text { Castro-Aguirre } \\
1978 .\end{array}$ & Varios años & $\begin{array}{l}\text { Todas las mencionadas } \\
\text { en esta columna; además } \\
\text { de anzuelo y línea de } \\
\text { mano y pesca comercial. }\end{array}$ & Esporádico & $\begin{array}{l}\text { Estuario del río } \\
\text { y canal de la laguna; } \\
\text { profundos y someros. }\end{array}$ & $\begin{array}{c}92^{3} \\
(51.4)\end{array}$ & 31 \\
\hline $\begin{array}{l}\text { Kobel-kowsky } \\
1985 .\end{array}$ & $\begin{array}{c}11 / 77 \\
a \\
02 / 79\end{array}$ & $\begin{array}{l}\text { Red de arrastre } \\
\text { Atarraya } \\
\text { Red de cuchara }\end{array}$ & $\begin{array}{l}6 \text { muestreos } \\
\text { sistemáticos } \\
\text { más algunas } \\
\text { recolectas } \\
\text { esporádicas. }\end{array}$ & $\begin{array}{l}\text { Diversos sitios } \\
\text { de la laguna; } \\
\text { profundidad variable. }\end{array}$ & $\begin{array}{c}68 \\
(38.0)\end{array}$ & 20 \\
\hline $\begin{array}{l}\text { Castro-Aguirre } \\
\text { et al. } 1986 .\end{array}$ & $\begin{array}{l}02 / 79 \\
a \\
09 / 80\end{array}$ & $\begin{array}{l}\text { Red de arrastre } \\
\text { (boca } 6.0 \mathrm{~m}, \phi 25 \mathrm{~mm} \text { ) } \\
\text { Ocasionalmente: } \\
\text { Chinchorro playero }\end{array}$ & $\begin{array}{l}\text { Bimestral } \\
9 \text { muestreos } \\
7 \text { estaciones }\end{array}$ & $\begin{array}{l}\text { Estuario del río y canal } \\
\text { de la laguna; profundos. } \\
\text { Lances ocasionales } \\
\text { en áreas someras cerca } \\
\text { de la boca del río. }\end{array}$ & $\begin{array}{c}99 \\
(55.3)\end{array}$ & 32 \\
\hline $\begin{array}{l}\text { Sánchez-Rueda } \\
1986 .\end{array}$ & $\begin{array}{c}10 / 83 \\
\text { a } \\
09 / 84\end{array}$ & $\begin{array}{l}\text { Red de arrastre } \\
\text { (boca } 5.5 \mathrm{~m}, \phi 25 \mathrm{~mm} \text { ) }\end{array}$ & $\begin{array}{l}\text { Mensual } \\
12 \text { muestreos } \\
3 \text { estaciones }\end{array}$ & $\begin{array}{l}\text { Estuario del río y } \\
\text { canal de la laguna; } \\
\text { profundos. }\end{array}$ & $\begin{array}{c}76 \\
(42.5)\end{array}$ & 10 \\
\hline $\begin{array}{l}\text { López-López } \\
\text { et al. } 1991\end{array}$ & $\begin{array}{c}.03 / 85 \\
\mathrm{a} \\
01 / 86\end{array}$ & $\begin{array}{l}\text { Red de arrastre } \\
\text { (boca } 2.0 \mathrm{~m}, \phi 20 \mathrm{~mm}) \\
\text { Chinchorro }(50 \mathrm{~m}, \phi 20 \mathrm{~mm} \text { ) }\end{array}$ & $\begin{array}{l}\text { Bimestral } \\
6 \text { muestreos } \\
15 \text { estaciones }\end{array}$ & $\begin{array}{l}\text { Diversos sitios de la } \\
\text { laguna, incluidas las } \\
\text { áreas someras }\end{array}$ & $\begin{array}{c}58 \\
(32.4)\end{array}$ & 6 \\
\hline $\begin{array}{l}\text { Cota y Santiago } \\
1994 .\end{array}$ & $\begin{array}{l}10 / 91 \\
a \\
09 / 92\end{array}$ & $\begin{array}{l}\text { Red de arrastre } \\
\text { (boca } 6.25 \mathrm{~m}, \phi 23 \mathrm{~mm}) \\
\text { Ocasionalmente: } \\
\text { Chinchorro }(50 \mathrm{~m}, \phi 25 \mathrm{~mm}) \\
\text { Agallera }(150 \mathrm{~m}, \phi 62 \mathrm{~mm}) \\
\text { Atarraya }(\phi 25 \mathrm{~mm})\end{array}$ & $\begin{array}{l}\text { Mensual } \\
11 \text { muestreos } \\
3 \text { estaciones } \\
22 \text { arrastres }\end{array}$ & $\begin{array}{l}\text { Canal de la laguna y } \\
\text { boca del río. } \\
\text { Lances ocasionales } \\
\text { en áreas someras cerca } \\
\text { de la boca del río }\end{array}$ & $\begin{array}{c}61 \\
(34.1)\end{array}$ & 5 \\
\hline Este trabajo & $\begin{array}{c}05 / 90 \\
\text { a } \\
06 / 91\end{array}$ & $\begin{array}{l}\text { Chinchorro } \\
(60 \mathrm{~m}, \phi 10 \mathrm{~mm})\end{array}$ & $\begin{array}{l}\text { Mensual } \\
13 \text { muestreos } \\
5 \text { estaciones }\end{array}$ & $\begin{array}{l}\text { Áreas someras con } \\
\text { vegetación sumergida } \\
\text { de la margen poniente } \\
\text { de la laguna. }\end{array}$ & $\begin{array}{c}85 \\
(47.5)\end{array}$ & 14 \\
\hline
\end{tabular}

1 Se refiere al porcentaje del total conbinado de 179 especies.

2 Se parte de la suposición de que los registros de Chávez (1972) son los primeros para el área.

3 No se consideran los registros de la desmbocadura del río ni aquellos consignados con base en comunicaciones personales, salvo cuando su presencia dentro del sistema fue posteriormente comprobada por otros autores. 


\section{RESULTADOS}

Al combinar los distintos listados, el número de especies de peces conocidas en el sistema estuarino-lagunar de Tuxpan-Tampamachoco asciende a 179 (Apéndice 1). Cabe señalar que este inventario, que sigue el arreglo propuesto por Nelson (1994), no incluye a 10 especies citadas por Castro-Aguirre $(1978,1986)$ para el área de Tuxpan, pues dichos registros aparentemente proceden de colectas efectuadas en el litoral marino adyacente, y no del interior del sistema estuarino-lagunar (éstas fueron los elasmobranquios Rhizoprionodon terranovae, Sphyrna lewini, Pristis perotteti, Dasyatis americana y Aetobatus narinari, y los peces óseos Urophycis floridanus, Floridichthys carpio,
Epinephelus adscensionis, Labrisomus nuchipinnis, y Microdesmus carri).

Cuando se comparan las distintas listas que conforman el inventario combinado se observa que sólo 22 especies (12.3\% del total) constituyen registros comunes a todos los trabajos; en contraste, 58 especies (32.4\%) sólo son mencionadas en uno de éstos. En lo que se refiere a la contribución de cada trabajo al número total de especies conocidas, el porcentaje más alto correspondió a Castro-Aguirre et al. (1986) y Castro-Aguirre (1986) y el más bajo a López-López et al. (1991) (Cuadro 1). Los valores del índice de similitud de Jaccard, que se obtuvieron al comparar la presencia, la ausencia y los registros comunes de especies en los distintos listados, variaron entre 33.7 y 66.3 (Cuadro 2).

\section{CUADRO 2}

Indices de similitud de Jaccard obtenidos al comparar los distintos listados de peces disponibles para el sistema estuarino lagunar de Tuxpan-Tampamachoco.

Table 2. Jaccard's similarity indexes obtained by comparing the fish species inventories available for the TuxpanTampamachoco estuarine system.

\begin{tabular}{|c|c|c|c|c|c|c|c|c|}
\hline & $\mathrm{a}$ & b & c & $\mathrm{d}$ & $\mathrm{e}$ & f & $\mathrm{g}$ & $\mathrm{h}$ \\
\hline $\mathbf{a}$ & 1 & 66.3 & 43.3 & 43.7 & 45.7 & 40.0 & 45.2 & 37.7 \\
\hline b & & 1 & 39.1 & 45.8 & 42.4 & 38.4 & 37.8 & 39.4 \\
\hline c & & & 1 & 45.2 & 41.2 & 38.5 & 57.3 & 48.5 \\
\hline d & & & & 1 & 50.9 & 36.5 & 45.4 & 42.6 \\
\hline e & & & & & 1 & 41.0 & 48.9 & 36.4 \\
\hline $\mathrm{f}$ & & & & & & 1 & 28.8 & 33.7 \\
\hline g & & & & & & & 1 & 37.7 \\
\hline h & & & & & & & & 1 \\
\hline
\end{tabular}

a) Chávez (1972), b) Castro-Aguirre (1978), c) Kobelkowsky (1985), d) Castro-Aguirre et al. y Castro-Aguirre (1986), e) Sánchez-Rueda (1986), f) López-López, et al. (1991), g) Cota y Santiago (1994) y h) este trabajo.

Un aspecto relevante de estos resultados lo constituye el hecho de encontrar una riqueza tan alta en un sistema tan pequeño (apenas 1500 ha), pues Reséndez y Kobelkowsky (1991) sugirieron la existencia de una relación directa entre la extensión de una laguna costera y su riqueza íctica. Para explorar numéricamente la validez de esta propuesta se evaluó, mediante un análisis de correlación simple, la relación existente entre la riqueza de especies en las 13 principales lagunas del Golfo de México y su área superficial (Fig. 1; Cuadro 3). Además de que el coeficiente de correlación obtenido fue bajo $(\mathrm{r}=0.54$; $\mathrm{p}<0.03)$, se observó que al eliminar del análisis a las lagunas más grandes (Madre, Tamiahua y Términos) la relación prácticamente desaparecía $(r=-0.06 ; p>0.43)$. Posteriormente, partiendo de la premisa de que las diferencias que actualmente se reconocen en la riqueza 
íctica de las lagunas costeras mexicanas podrían deberse en gran parte a diferencias importantes en la intensidad de muestreo a la que han sido sometidas (Torres-Orozco 1994), se relacionó del mismo modo la riqueza de especies con el número de trabajos ictiológicos y de inventarios ictiofaunísticos, respectivamente, realizados en cada laguna (Cuadro 3). En el primer caso, el coeficiente de correla- ción obtenido fue mucho mayor $(\mathrm{r}=0.77$; $\mathrm{p}=$ 0.001 ) y aumentó todavía más cuando se consideraron únicamente los inventarios $(\mathrm{r}=0.92$; $\mathrm{p}=0.000$ ). En esta ocasión, al excluir del análisis a las lagunas más grandes (Madre, Tamiahua y Términos) la relación con el número de trabajos ictiológicos disminuyó ( $\mathrm{r}=$ $0.57 ; \mathrm{p}<0.05$ ), pero aquella con el número de inventarios se mantuvo $(r=0.91 ; \mathrm{p}=0.000)$.

Cuadro 3

Principales lagunas costeras del Golfo de México, número de especies de peces registradas, superficie de las lagunas y número de trabajos de tema ictiológico, en general, y de inventarios ictiofaunísticos, en particular, realizados en cada laguna.

Table 3. Main Mexican coastal lagoons of the Gulf of Mexico, number of recorded fish species, areas of the lagoons and number of ichtiological studies and fish inventories known for each lagoon.

Sistema estuarino-lagunar

Laguna Madre, Tamps.

L. Pueblo Viejo, Ver.

L. de Tamiahua, Ver.

Sist. Tuxpan-Tampamachoco, Ver.

Laguna Grande, Ver.

L de la Mancha, Ver.

L. de Mandinga, Ver.

L. de Alvarado, Ver.

L. de Sontecomapan, Ver.

Laguna del Ostión, Ver.

Sist. Carmen-Machona, Tab.

L. de Mecoacán, Tab.

L. de Términos, Camp.

$\begin{array}{cr}\begin{array}{c}\text { No. de spp. } \\ \text { de peces }\end{array} & \text { Área }^{1} \text { (ha) } \\ \text { registradas } & \\ & \\ 105^{3} & 200,000 \\ 67^{4} & 9,300 \\ 112^{5} & 88,000 \\ 179^{6} & 1,500 \\ 40^{3} & 2,250 \\ 60^{7} & 140 \\ 88^{8} & 3,250 \\ 89^{3} & 6,200 \\ 97^{9} & 891 \\ 37^{10} & 1,270 \\ 81^{3} & 15,300 \\ 49^{11} & 5,168 \\ 216^{3} & 170,000\end{array}$

\section{No. de estudios ictiológicos ${ }^{2}$}

No. de inventarios de ictiofauna ${ }^{2}$

$\begin{array}{rr}4 & 3 \\ 32 & 4 \\ 39 & 11 \\ 45 & 15 \\ 7 & 0 \\ 14 & 6 \\ 9 & 4 \\ 59 & 5 \\ 8 & 4 \\ 4 & 2 \\ 5 & 3 \\ 2 & 2 \\ 93 & 23\end{array}$

Notas: ' Contreras, 1993; ${ }^{2}$ Información recabada en el Centro de Documentación Sobre Ecosistemas Litorales Mexicanos (Universidad Autónoma Metropolitana - Iztapalapa, México, D.F.), cuyos registros incluyen tanto trabajos publicados como comunicaciones presentadas en congresos. ${ }^{3}$ Reséndez y Kobelkowsky 1991; ${ }^{4}$ Castillo $1995 ;{ }^{5}$ Franco-López y ChávezLópez 1992; ${ }^{6}$ Este trabajo; ${ }^{7}$ Martínez et al. 1995; ${ }^{8}$ De la Cruz et al. 1985; ${ }^{9}$ Fuentes et al. 1989; ${ }^{10}$ Bozada et al. 1982; 11 Granados et al. 1992.

En las altas correlaciones de la riqueza de especies con el número de inventarios no sólo está implícito un mayor esfuerzo de captura, sino una más amplia variedad en las estrategias de recolecta. Cuando se elabora un gráfico del número acumulativo de especies registradas por primera vez en el sistema de Tuxpan-Tampamachoco a través del tiempo, la curva resultante se caracteriza por presentar en su prime- ra parte una elevada pendiente, en la que cada nuevo estudio añade un promedio de 28 registros a la lista de especies (Fig. 2). Como puede apreciarse en el Cuadro 1, esta parte de la curva se relaciona con el empleo de artes de pesca distintas de la red de arrastre en cada trabajo consecutivo. Así, en los tres trabajos siguientes, que fueron realizados con una estrategia de muestreo similar a la de los anteriores 
(i. e. empleando las mismas artes en áreas semejantes), el número de registros nuevos disminuye paulatinamente y la curva denota una tendencia a alcanzar una asíntota; sin embargo, al intensificar el muestreo en las zonas con pastos marinos empleando un chinchorro de malla fina (nuestras recolectas) la pendiente se incrementa de nuevo.

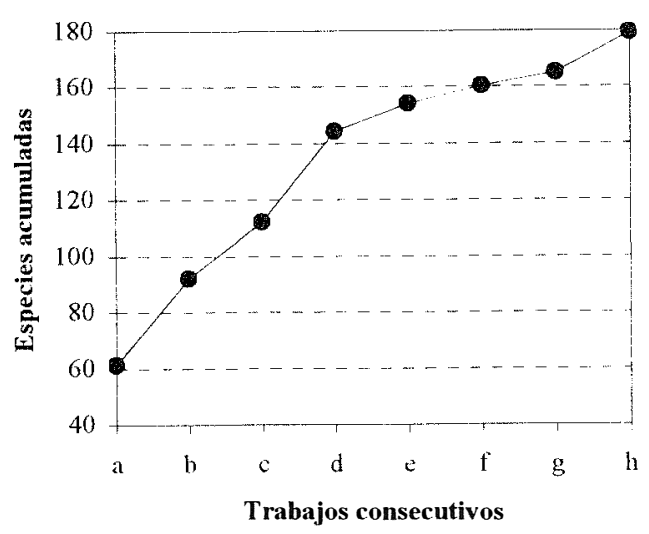

Fig. 2. Incremento en el número de especies de peces conocidas para el sistema estuarino-lagunar de TuxpanTampamacho en función de los esfuerzos de recolecta consecutivos. Las letras a-h se refieren a los distintos trabajos realizados en el área (ver Apéndice. 1), ordenados cronológicamente.

Fig. 2. Increment in the number of fish species known for the Tuxpan-Tampamacho estuarine system as a function of the consecutive sampling efforts. Letters a-h refers to different studies made in this area (see Appendix 1), chronologically ordered.

\section{DISCUSIÓN}

Las 179 especies de peces que conforman la ictiofauna conocida hasta la fecha para el sistema Tuxpan-Tampamachoco equivalen al $56.3 \%$ del total de las especies citadas por Reséndez y Kobelkowsky (1991) para las 13 lagunas costeras más conspicuas del Golfo de México (Fig. 1). En virtud de lo anterior, y tomando en cuenta el estado actual del conocimiento de la ictiofauna estuarina mexicana, este ambiente se destaca como uno de los más ricos en especies de peces de las costas del Atlántico de México, en donde sólo es superado por la Laguna de Términos (Fuentes-Mata, 1991).

La baja similitud entre los listados de especies de peces disponibles para el sistema Tuxpan-Tampamachoco puede atribuirse, fundamentalmente, a dos causas: 1) a las diferencias en las estrategias de recolección, que incluyen: a) el uso de artes de pesca de distinto tipo y selectividad; b) la ubicación de los sitios de recolecta con respecto a los distintos microhábitats presentes en el área, y c) las diferencias en la intensidad de las recolectas - duración de los períodos de muestreo, distribución temporal y frecuencia de las recolectas, número de estaciones, número de lances, tamaño de las superficies de barrido, etc.-, ó 2) a la existencia de cambios temporales en la composición, distribución y abundancia de las asociaciones de peces, derivados de cambios naturales o inducidos en la dinámica ambiental. $\mathrm{La}$ variedad de métodos utilizados en los distintos trabajos apoya la primera hipótesis.

Las especies comunes a todos los trabajos parecen ser las más abundantes y de más amplia distribución en el sistema; por su parte, la gran cantidad de registros únicos podría deberse a que dichas especies: 1) son poco abundantes, 2) tienen una distribución restringida a ciertos microhábitats o, 3) son estrictamente dulceacuícolas o marinas estenohalinas, por lo que su presencia en el ambiente estuarino es accidental.

La baja correlación observada entre el área de las lagunas y su riqueza de especies no implica necesariamente que la relación especies - área no se cumpla para el caso de los peces de los sistemas estuarino-lagunares; sin embargo, aunque evaluar la validez de tal relación rebasa los objetivos de este trabajo, nuestros resultados sugieren que la relación propuesta por Reséndez y Kobelkowsky (1991) podría ser un artificio derivado del hecho de que las lagunas costeras grandes han sido, en general, más estudiadas que las pequeñas.

Nuestras recolectas en los ceibadales de Tampamachoco brindaron 14 especies de peces cuya presencia en la laguna no había sido previamente documentada. Estos hallazgos nos 
permiten suponer que algo semejante podría esperarse de las áreas pantanosas de mayor influencia continental de la parte noroccidental de la laguna, hasta ahora muy poco estudiadas. Fuentes-Mata (1991) ha señalado que la riqueza de peces de los sistemas estuarino-lagunares de México se distingue por una marcada dominancia de elementos de origen marino ( $\approx 50$ especies de un total de 400), lo cual, según Castro-Aguirre (1978), se deriva de la pobreza del componente dulceacuícola primario de la ictiofauna mexicana. Pese a ello, es muy probable que el componente dulceacuícola de la ictiofauna estuarino-lagunar de México sea más importante de lo que se ha estimado. Un mayor esfuerzo de captura con artes de pesca diversas y adecuadas en los distintos microhábitats lagunares podría aumentar notablemente nuestro conocimiento de su riqueza íctica.

El gran número de especies registradas en el área de estudio, relativamente pequeña, sugiere que el conocimiento de la riqueza íctica de la gran mayoría de los sistemas estuarino-lagunares de México es todavía incompleto. Ya que las principales limitaciones para integrar listas de especies razonablemente completas son de índole metodológica, conviene insistir en que para el estudio de la ictiofauna de los sistemas estuarino-lagunares es fundamental un diseño cuidadoso de las estrategias de recolección, en el cual debe procurarse: a) emplear para las capturas la mayor variedad posible de artes de pesca, b) recolectar en los distintos microhábitats identificables dentro del cuerpo lagunar y, c) con el fin de poder detectar las posibles variaciones interanuales en la composición y estructura de los ensambles ícticos, preferir los muestreos cada dos o tres meses por dos o más años sobre los muestreos mensuales durante un sólo año.

\section{RESUMEN}

Se analiza la manera como se ha conformado el conocimiento de la ictiofauna del sistema estuarino-lagunar de Tuxpan-Tampamachoco, en el estado mexicano de Veracruz. Se elaboró un inventario de los peces cono- cidos hasta la fecha en dicho sistema $(\mathrm{N}=179)$, a partir de listas previamente publicadas y de muestreos propios en las áreas someras de la laguna cubiertas por pastos marinos, que nos permitieron detectar 14 especies antes desconocidas en el área. Al comparar los distintos listados se observa una escasa similitud, la cual se atribuye a las diferencias en las estrategias de recolecta empleadas. $\mathrm{Al}$ menos con los datos actualmente disponibles, la riqueza íctica registrada en las lagunas costeras del Golfo de México no está relacionada con su área, como se ha sugerido, sino con el número de inventarios disponibles para cada laguna, pues éste involucra importantes diferencias en el esfuerzo de muestreo y en las estrategias de recolecta. Se recomienda un diseño de muestreo que contemple: a) emplear la mayor variedad posible de artes de pesca, b) recolectar en todos los tipos de microhábitat y, c) preferir los muestreos cada dos o tres meses por dos o más años sobre los muestreos mensuales durante un sólo año.

\section{REFERENCIAS}

Berry, F. H. 1978. Carangidae. In W. Fischer, (ed.). FAO Species Identification Sheets for Fishery Purposes. Western Central Atlantic. (fishing area 31). Vols. I \& II. FAO. Roma.

Bozada R., L. M., F. Cancino C., Z. Chávez A. \& J. A. García N. 1982.Análisis de la comunidad del sistema lagunar del Ostión, Veracruz, México. Resúmenes. II Congreso sobre Problemas Ambientales de México: 17.

Bradbury, M. G. 1980. A revision of the fish genus Ogcocephalus with descriptions of new species from the Western Atlantic Ocean (Ogcocephalidae: Lophiiformes). Proc. Calif. Acad. Sci. 42: 229-285.

Castillo R., M. 1995. Aspectos ecológicos de la ictiofauna de la laguna de Pueblo Viejo, Veracruz. Tesis de Maestría. Facultad de Ciencias. Universidad Nacional Autónoma de México, México, D.F. 96 p.

Castro-Aguirre, J. L. 1978. Catálogo sistemático de los peces marinos que penetran a las aguas continentales de México, con aspectos zoogeográficos y ecológicos. Departamento de Pesca, Dirección General del Instituto Nacional de la Pesca, México, D.F. Serie Científica No. 19: 1-298.

Castro-Aguirre, J. L. 1986. Estudios sistemáticos y ecológicos de la ictiofauna del sistema estuarino-lagunar Tuxpan-Tampamachoco, Veracruz, México. Tesis Doctoral. Escuela Nacional de Ciencias Biológicas, Instituto Politécnico Nacional, México, D.F. 233 p. 
Castro-Aguirre, J. L., R. Torres-Orozco B., M. Ugarte A. \& A. Jiménez. 1986. Estudios ictiológicos en el sistema estuarino-lagunar Tuxpan-Tampamachoco, Veracruz. I. Aspectos ecológicos y elenco sistemático. An. Esc. Nal. Cienc. Biol., IPN, México, 30: 155-170.

Chávez, E. A. 1972. Notas acerca de la ictiofauna del estuario del Río Tuxpan y sus relaciones con la temperatura y la salinidad. p. 177-199. In J. Carranza (ed). Memorias del IV Congreso Nacional de Oceanografía. 17-19 de noviembre de 1969, México, D.F.

Contreras, F. 1993. Ecosistemas costeros mexicanos. CONABIO-UAMI, México, D.F. 415 p.

Cota, F. V. \& R. Santiago B. 1994. Estudio de la estructura de las comunidades de peces de la laguna de Tampamachoco, Veracruz. Oceanología 1:149-173.

Deckert, D.G. \& D. W. Greenfield. 1987. A review of the Western Atlantic species of the genera Diapterus and Eugerres (Pisces: Gerreidae). Copeia 1987: 182-194.

De la CruzA., G., J. Franco-López \& L. G. Abarca A. 1985. Caracterización ictiofaunística de los sistemas estuarinos del estado de Veracruz, México. Memorias del VII Congreso Nacional de Zoología: 175-187.

Franco-López, J. \& R. Chávez-López. 1992. Síntesis sobre el conocimiento de la ictiofauna de la laguna de Tamiahua, Veracruz, México. Hidrobiológica 3/4: 53-63.

Fuentes M., P., H. Espinosa P. \& J. Luna W. 1989. Nuevos registros de peces en la laguna de Sontecomapan, Veracruz, México. An. Inst. Biol. Univ. Nal. Autón. México. Serie Zoología 60: 257-262.

Fuentes-Mata, P. 1991. Diversidad ictiofaunística en sistemas lagunares de México, p. 66-73. In M. G Figueroa, C. Álvarez, A. Esquivel \& M. E. Ponce (eds.). Físico-química y biología de las lagunas costeras mexicanas. DCBS, Universidad Autónoma Metropolitana-Iztapalapa, México, D.F. Serie Grandes Temas de la Hidrobiología 1.

Granados A., A., M. Priego, C. Salvadores \& J. L. Ramos. 1992. Fauna acuática de la Laguna de Mecoacán, Paraíso, Tabasco. Resúmenes del IX Congreso Nacional de Oceanografía: 99.
Hoese, H. D. \& R. H. Moore. 1977. Fishes of the Gulf of Mexico: Texas, Louisiana and adjacent waters. Texas University Press. College Station and London. $327 \mathrm{p}$.

Kobelkowsky D., A. 1985. Los peces de la laguna de Tampamachoco, Veracruz, México. Biótica 10:145-156.

López-López, E., M. Salgado-Mejía \& S. A. Guzmán del Proo. 1991. Un análisis estacional de la ictiofauna de la laguna de Tampamachoco, Ver., y sus hábitos alimentarios. An. Esc. Nal. Cienc. Biol., IPN, México 34: 81-107.

Martínez T., M. E., N. B. Ramírez L. \& J. Rojas G. 1995. Análisis comparativo de los componentes ictiofaunísticos de la laguna de la Mancha, Veracruz, en los últimos veinte años. Resúmenes del XIII Congreso Nacional de Zoología: 78.

Nelson, J. S. 1994. Fishes of the world. Wiley, Nueva York. $600 \mathrm{p}$.

Reséndez, A. \& A. Kobelkowsky. 1991. Ictiofauna de los sistemas lagunares costeros del Golfo de México, México. Universidad y Ciencia 8: 91-110.

Sánchez-Rueda, M. P. 1986. Contribución al conocimiento de la ictiofauna del sistema estuarino-lagunar Tuxpam-Tampamachoco, Veracruz, México (19831984). Tesis profesional. Universidad Autónoma de Baja California Sur. Area de Ciencias del Mar, Departamento de Biología Marina. La Paz, B.C.S., México. $102 \mathrm{p}$.

Torres-Orozco B., R. 1994. Los peces, p. 270-304. In G. De la Lanza E. \& C. Cáceres M. (eds.). Lagunas costeras y el litoral mexicano. Universidad Autónoma de Baja California Sur, La Paz, B.C.S., México.

Whitehead, P. J. P., G. J. Nelson \& T. Wongratana. 1988. FAO species catalogue. Clupeoid fishes of the World. An annotated and ilustrated catalogue of the herrings, sardines, pilchards, sprads, anchovies and wolfherrings. Part 2. Engraulidae. FAO Fish. Synop. 7(125): 1-303. 


\section{Apéndice 1}

Peces registrados en el sistema estuarino-lagunar de Tuxpan-Tampamachoco por distintos autores.

Appendix 1. Fish species recorded by different authors in the Tuxpan-Tampamachoco estuarine system.

\section{Especie}

\section{CHONDRICHTHYES}

Carcharhiniformes

Carcharhinidae

Negaprion brevirostris (Poey)

Carcharhinus porosus (Ranzani)*

Carcharhinus leucas (Valenciennes)

Sphyrna tiburo (Linnaeus)

Sphyrna mokarran (Ruepell)

\section{Rajiformes}

Narcinidae

Narcine brasiliensis (Olfers)

Rhinobatidae

Rhinobatos lentiginosus (Garman)

Rajidae

Raja texana Chandler

Dasyatidae

Dasyatis sabina (Lesueur)

Gymnuridae

Gymnura micrura (Bloch y Schneider)

TELEOSTOMI

\section{Elopiformes}

Elopidae

Elops saurus Linnaeus

Megalopidae

Megalops atlanticus Valenciennes

Albuliformes

Albulidae

Albula vulpes (Linnaeus)

Anguilliformes

Muraenidae

Gymnothorax nigromarginatus (Girard)

Ophichthidae

Ophichthus gomesii (Castelnau)

Myrophis punctatus Lutken

Clupeiformes

Engraulidae

Anchoa hepsetus (Linnaeus)

Anchoa mitchilli (Valenciennes)

Anchoa lyolepis (Evermann y Marsh)

Anchoa cubana (Poey)

Anchoa lamprotaenia Hildebrand

Cetengraulis edentulus (Cuvier) a

b

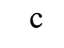

d

e f g

$\mathrm{h}$

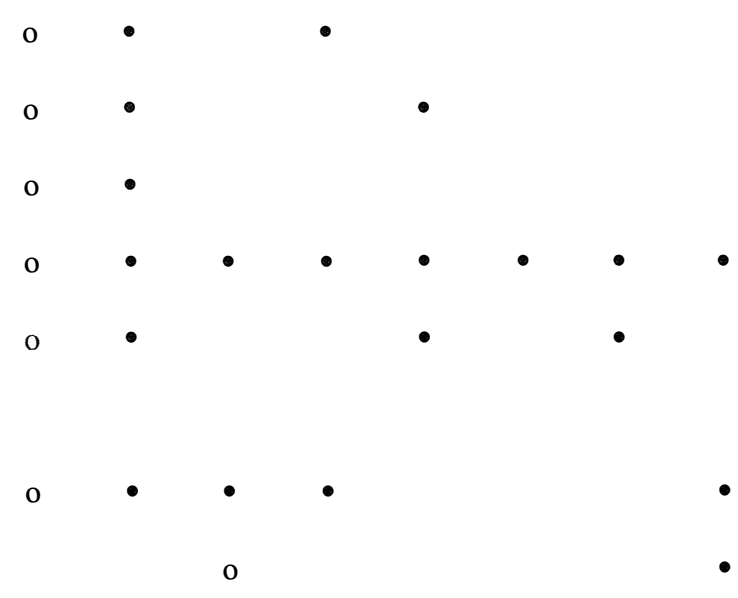

o

\section{o}
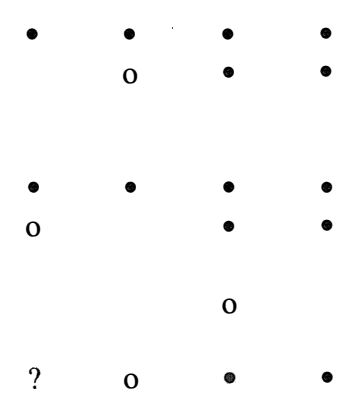
Especie

Clupeidae

Harengula jaguana Poey

Harengula clupeola (Cuvier)

Opistonema oglinum (Lesueur)

Sardinella aurita Valenciennes

Brevoortia patronus Goode

Brevoortia guntheri Hildebrand

Dorosoma petenense (Gunther)

Siluriformes

Ariidae

Bagre marinus (Mitchill)

Ariopsis felis (Linnaeus)

Cathorops melanopus (Gunther)

Aulopiformes

Synodontidae

Synodus foetens (Linnaus)

Ophidiiformes

Ophidiidae

Brotula barbata (Bloch)

Batrachoidiformes

Batrachoididae

Porichthys plectrodon Jordan y Gilbert

Opsanus beta (Goode y Bean)

Lophiiformes

Antennariidae

Antennarius tenebrosus (Poey)

Histrio histrio (Linnaeus)

Ogcocephalidae

Ogcocephalus cubifrons (Richardson)

Ogcocephalus pantosticus Bradbury

Mugiliformes

Mugilidae

Mugil cephalus Linnaeus

Mugil curema Valenciennes

Mugil trichodon Poey

Agonostomus monticola (Banchroft)

Atheriniformes

Atherinidae

Membras martinica (Valenciennes)

Menidia beryllina Günther

Beloniformes

Belonidae

Strongylura marina (Walbaum)

Strongylua timucu (Walbaum)

Strongylura notata (Poey)

Hemiramphidae

Chriodorus atherinoides Goode y Bean Hemiramphus brasiliensis (Linnaeus)

Hyporhamphus unifasciatus (Ranzani)

Hyporhamphus roberti (Valenciennes) a

b

c d

$\mathrm{e}$

$\mathrm{h}$

O

$?$

-

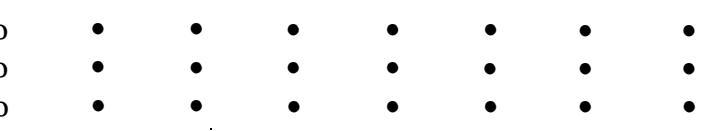

o

o

o

o

- $\quad \bullet$

0
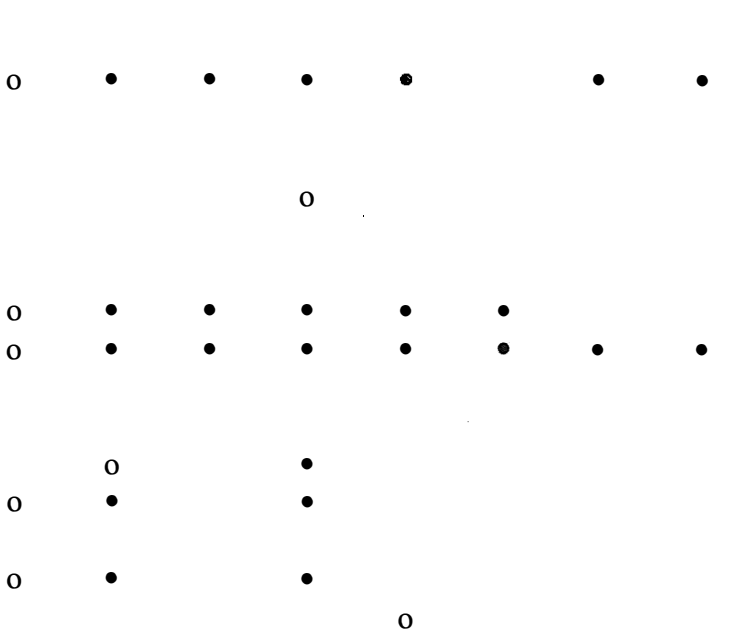

$\begin{array}{llllllll}0 & \bullet & \bullet & \bullet & & \bullet & \bullet & \bullet \\ 0 & \bullet & \bullet & \bullet & \bullet & \bullet & \bullet & \bullet \\ 0 & & & \bullet & & & \end{array}$

o

o

o

o

o

o

o

o 
Especie

\section{Cyprinodontiformes}

Fundulidae

Fundulus grandis Baird y Girard

Lucania parva (Baird y Girard)

Poeciliidae

Poecilia latipunctata Meek

Gambusia regani Hubbs

Cyprinodontidae

Cyprinodon variegatus Lacépede

\section{Gasterosteiformes}

Syngnathidae

Syngnathus louisianae Günther

Syngnathus floridae (Jordan y Gilbert)

Syngnathus scovelli (Evermann y Kendall)

Oostethus lineatus (Kaup)

Hippocampus erectus Perry

Scorpaeniformes

\section{Scorpaenidae}

Scorpaena plumieri Bloch

Scorpaena dispar Longley y Hildebrand

Triglidae

Prionotus tribulus (Cuvier)

Prionotus punctatus (Bloch)

\section{Perciformes}

\section{Centropomidae}

Centropomus parallelus Poey

Centropomus undecimalis (Bloch)

Centropomus poeyi Chávez

Centropomus pectinatus Poey

Centropomus ensiferus Poey

Serranidae

Serranus atrobranchus (Cuvier)

Epinephelus striatus (Bloch)

Epinephelus guttatus (Linnaeus)

Epinephelus nigritus (Holbrook)

Mycteroperca bonaci (Poey)

Rypticus saponaceus (Bloch y Schneider)

Pomatomidae

Pomatomus saltatrix (Linnaeus)

Echeneidae

Echeneis naucrates Linnaeus

Carangidae

Oligoplites saurus (Bloch y Schneider)

Trachinotus carolinus (Linnaeus)

Trachinotus falcatus (Linnaeus)

Chloroscombrus chrysurus (Linnaeus)

Selene vomer (Linnaeus)

Selene setapinnis (Mitchill)

Caranx hippos (Linnaeus)

Caranx latus Agassiz 
Especie

Caranx crysos (Mitchill)

Hemicaranx amblyrhynchus (Val.)

Selar crumenophtalmus (Bloch)

Lutjanidae

Lutjanus synagris (Linnaeus)

Lutjanus griseus (Linnaeus)

Lutjanus jocu (Bloch y Schneider)

Lutjanus analis (Cuvier y Valenciennes)

Lutjanus cyanopterus (Cuvier)

Lobotidae

Lobotes surinamensis (Bloch)

Gerreidae

Eugerres plumieri (Cuvier)

Gerres cinereus (Walbaum)

Eucinostomus argenteus (Baird y Girard)

Eucinostomus melanopterus (Bleeker)

Eucinostomus gula (Cuvier)

Diapterus auratus Ranzani

Diapterus rhombeus (Cuvier)

Ulaema lefroyi (Goode)

Haemulidae

Conodon nobilis (Linnaeus)

Pomadasys crocro (Valenciennes)

Orthopristis chrysoptera (Linnaeus)

Anisotremus surinamensis (Bloch)

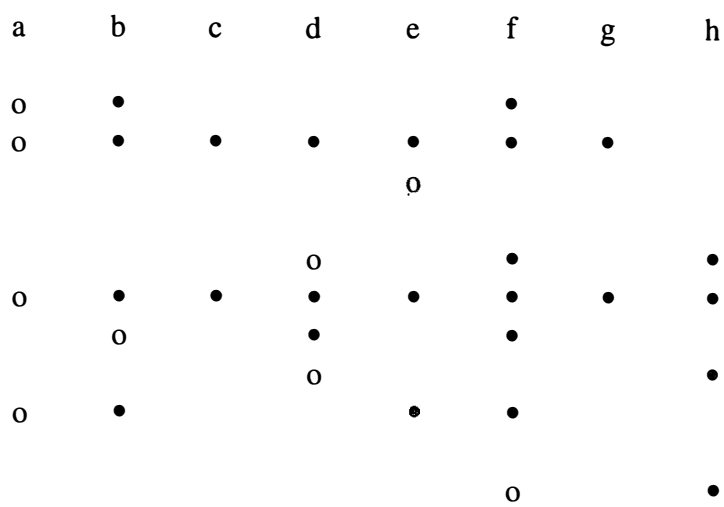

Haemulon bonariense (Cuvier)

o

Haemulon sciurus (Shaw)

Sparidae

Stenotomus chrysops (Linnaeus)

Archosargus rhomboidalis (Linnaeus)

Archosargus probatocephalus (Walbaum)

Lagodon rhomboides (Linnaeus)

Polynemidae

Polydactylus octonemus (Girard)

Sciaenidae

Cynoscion arenarius Ginsburg

Cynoscion nothus (Holbrook)

Cynoscion nebulosus (Cuvier)

Cynoscion jamaicensis (Vaillant y Bocourt)

Pogonias cromis (Linnaeus)

Micropogonias undulatus (Linnaeus)

Micropogonias furnieri (Desmatrest)

Menticirrhus littoralis (Holbrook)

Menticirrhus americanus (Linnaeus)

Menticirrhus saxatilis (Bloch y Schneider)

Umbrina coroides Cuvier

Umbrina broussonetii Cuvier

Leiostomus xanthurus Lacépede

Sciaenops ocellata (Linnaeus)

Stellifer lanceolatus (Holbrook) 
Especie

Stellifer stellifer (Bloch)

Larimus fasciatus Holbrook

Bairdiella chrysoura (Lacépede)

Bairdiella ronchus (Cuvier)

Chaetodontidae

Chaetodon ocellatus Bloch

Cichlidae

Herichthys cyanoguttatus (Baird y Girard)

Uranoscopidae

Astroscopus y-graecum (Cuvier)

Labrisomidae

Labrisomus guppyi (Norman)

Blenniidae

Blennius cristatus Linnaeus

Hypsoblennius henzi (LeSueur)

Semablennius nicholsi (Fowler)

Gobiesocidae

Gobiesox strumosus Cope

Eleotridae

Dormitator maculatus (Bloch)

Eleotris pisonis (Gmelin)

Erotelis smaragdus (Valenciennes)

Gobiomorus dormitor Lacépede

Gobiidae

Bathygobius soporator (Valenciennes)

Garmannia hemigymna (Eigen. y Eigen.)

Evorthodus lyricus (Girard)

Gobioides broussonnetti Lacépede

Gobiosoma bosci (Lacépede)

Gobionellus hastatus Girard

Gobionellus boleosoma (Jordan y Gilbert)

Gobionellus schufeldti (Jordan y Eigen.)

Lophiogobius cyprinoides (Pallas)

Ephippidae

Chaetodipterus faber (Broussonet)

Sphyraenidae

Sphyraena barracuda (Walbaum)

Sphyraena guachancho Cuvier

Trichiuridae

Trichiurus lepturus Linnaeus

Scombridae

Scomberomorus maculatus (Mitchill)

Stromateidae

Peprilus burti Fowler

Peprilus paru (Linnaeus)

Pleuronectiformes

Paralichthyidae

Etropus crossotus Jordan y Gilbert

Citharichthys spilopterus Günther

Citharichthys abboti Dawson a

$$
\text { b }
$$$$
\text { c }
$$

g h

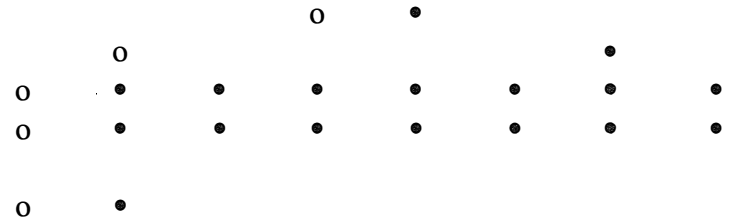

O o

o

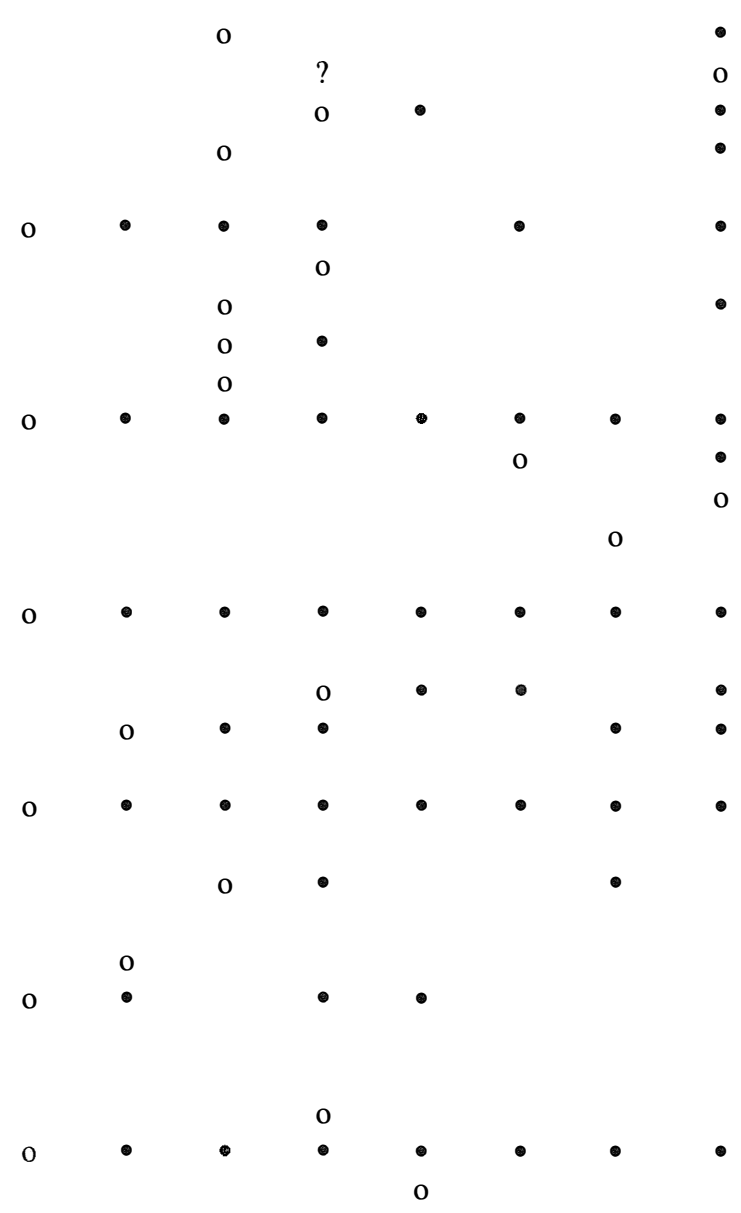


Especie

Citharichthys macrops Dressel

Achiridae

Achirus lineatus (Linnaeus)

Trinectes maculatus (Bloch y Schneider)

Gymnachirus texae (Günther)

Cynoglossidae

Symphurus plagiusa (Linnaeus)

Symphurus civitatum Ginsburg

Tetraodontiformes

Balistidae

Balistes capriscus Gmelin

Monacanthidae

Aluterus scriptus (Osbeck)

Stephanolepis hispidus (Linnaeus)

Tetraodontidae

Lagocephalus laevigatus (Linnaeus)

Sphoeroides parvus Shipp y Yerger *

Diodontidae

Diodon hystrix Linnaeus

Chilomycterus schoepfi (Walbaum)*

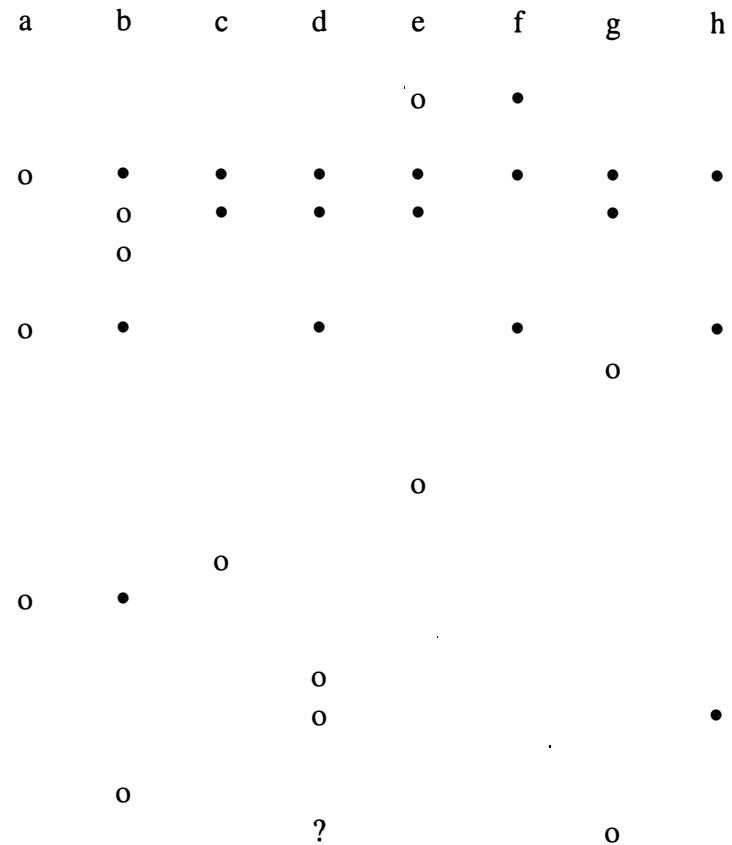

Notas: a) Chávez 1972, b) Castro-Aguirre 1978, c) Kobelkowsky 1985, d) Castro-Aguirre et al. 1986 y Castro-Aguirre 1986, e) Sánchez-Rueda 1986, f) López-López et al. 1991, g) Cota y Santiago 1994 y h) este trabajo. Con excepción de los registros de la columna b, que se basan en ejemplares obtenidos de recolectas aisladas y depositados en colecciones, los registros proceden de recolectas sistemáticas realizadas por los distintos autores.

El registro de Anchoa parva reportado en d con base en dos ejemplares de más de $71 \mathrm{~mm}$ de LP es dudoso pues según Whitehead et al. (1988) esta especie no rebasa los $6 \mathrm{~cm}$ de LP y se distribuye de Cuba a Jamaica y del oriente de Yucatán hasta Colombia. El registro de Membras vagrans de d se incluye aquí bajo el nombre de M. martinica, atendiendo a lo sugerido por Hoese y Moore (1977); Diapterus olisthostomus de a, b, y f, y D. evermani, de f, son sinónimos de D. auratus (Deckert y Greenfield 1987). Nautopaedium porosissimus de a es sinónimo de Porichthys porossisimus de c y f; sin embargo, todos estos registros son en realidad $P$. plectrodon, pues $P$. porosissimus se distribuye desde el sur de Brasil hasta Argentina (Castro-Aguirre 1986). Ogcocephalus vespertilio de a y O. parvus de b y d son sinónimos de O. cubifrons (Bradbury 1980). Achirus fasciatus de g se considera sinónimo de Trinectes maculatus (Castro-Aguirre 1978). Caranx fusus de a, b y f es sinónimo de Caranx crysos y Vomer setapinnis de a es sinónimo de Selene setapinnis (Berry 1977). Poronotus triacanthus de b, es sinónimo de Peprilus burti (Castro-Aguirre 1986). Larimus breviceps de b es sinónimo de L. fasciatus (Castro-Aguirre 1986). o = primer registro; * = Señala las únicas especies citadas por Castro-Aguirre (1986) que no fueron consignadas en trabajos previos (por esta razón se incluyen en la misma columna los registros de Castro-Aguirre et al. 1986 y Castro-Aguirre 1986). 